\title{
Fourier Hyperfunctions as the Boundary Values of Smooth Solutions of Heat Equations
}

\author{
By \\ Kwang Whoi KIM*, Soon-Yeong CHUNG** and Dohan $\mathrm{KIM}^{* * *}$
}

\begin{abstract}
We show that if a $C^{\infty}$-solution $u(x, t)$ of heat equation in $\boldsymbol{R}_{+}^{n+1}$ does not increase faster than $\exp \left[\varepsilon\left(\frac{1}{t}+|x|\right)\right]$ then its boundary value determines a unique Fourier hyperfunction. Also, we prove the decomposition theorem for the Fourier hyperfunctions. These results generalize the theorems of T. Kawai and T. Matsuzawa for Fourier hyperfunctions and solve a question given by A. Kaneko.
\end{abstract}

\section{§ 0. Introduction}

T. Kawai and T. Matsuzawa have shown in $[10,15]$ that the boundary value of a $C^{\infty}$-solution of heat equation in $\boldsymbol{R}_{+}^{n+1}$ which does not increase faster than $\exp (\varepsilon / t)$ is a well-defined hyperfunction. However, little is known about the characterization of a solution whose boundary value determines a Fourier hyperfunction near a characteristic boundary point. The purpose of this paper is to discuss this problem, that is, if a $C^{\infty}$-solution $U(x, t)$ satisfies some growth condition (see (2.2)) then we can assign a unique compactly supported Fourier hyperfunction $u(x)$ to $U(x, t)$. Furthermore we can find such a Fourier tame solution $U(x, t)$ of heat equation for any compactly supported Fourier hyperfunction $u(x)$. To show this, we use the estimate for the heat kernel in [15] and structure theorems of ultradistributions given in [11, 13].

We use the multi-index notations such as $|\alpha|=\alpha_{1}+\cdots+\alpha_{n}, \partial^{\alpha}=\partial_{1}^{\alpha} \partial_{2}^{\alpha}{ }_{2} \ldots$ $\partial_{n}^{\alpha_{n}}, \partial_{j}=\partial / \partial x_{j}$ for $\alpha=\left(\alpha_{1}, \alpha_{2}, \cdots, \alpha_{n}\right) \in N_{0}^{n}$ where $N_{0}$ is the set of nonnegative

Communicated by T. Kawai, February 10, 1992. Revised March 30 and July 6, 1992. 1991 Mathematics Subject Classification: 46F20, 46F15, 35K05

* Department of Mathematics Education, Jeonju University, Jeonju 560-759, Korea

** Department of Mathematics, Duksung Women's University, Seoul 132-714, Korea

*** Department of Mathematics, Seoul National University, Seoul 151-742, Korea Research partially supported by KOSEF and GARC. 
integers, and $\partial_{t}=\partial / \partial t$

\section{$\S 1$. Complex and Real Versions of Fourier Hyperfunctions}

First, we are going to introduce the complex and real versions of Fourier hyperfunctions and show their equivalence.

We denote by $\mathbb{D}^{n}$ the compactification $\mathbb{R}^{n} \cup S_{\infty}^{n-1}$ of $\mathbb{R}^{n}$, where $S_{\infty}^{n-1}$ is an $(n-1)$-dimensional sphere at infinity. When $x$ is a vector in $\mathbb{R}^{n} \backslash\{0\}$, we denote by $x \infty$ the point on $S^{n-1}$ which is represented by $x$, where we identify $S^{n-1}$ with $\mathbb{R}^{n} \backslash\{0\} / \mathbb{R}^{+}$. The space $\mathbb{D}^{n}$ is given the natural topology, that is: (i) If a point $x$ of $\mathbb{D}^{n}$ belongs to $\mathbb{R}^{n}$, a fundamental system of neighborhoods of $x$ is the set of all open balls containing the point $x$. (ii) If a point $x \in \mathbb{D}^{n}$ belongs to $S_{\infty}^{n-1}$, a fundamental system of neighborhoods of $x(=y \infty)$ is given by the following family

$$
U_{\tilde{\Delta}, A}(y \infty)=\left\{x \in \mathbb{R}^{n} ; x /|x| \in \tilde{\Delta},|x|>A\right\} \cup\{a \infty ; a \in \widetilde{\Delta}\},
$$

where $\tilde{\Delta}$ is a neighborhood of $y$ in $S^{n-1}$.

Definition 1.1. Let $K$ be a compact set in $\mathbb{D}^{n}$. We say that $\phi$ is in $\mathscr{F}(K)$ if $\phi \in C^{\infty}\left(\Omega \cap \mathbb{R}^{n}\right)$ for any neighborhood $\Omega$ of $K$ and if there are positive constants $h$ and $k$ such that

$$
|\phi|_{k, h}=\sup _{x \in \Omega \bigcap_{\alpha} \mathbb{R}^{n}} \frac{\left|\partial^{\alpha} \phi(x)\right|}{h^{|\alpha|} \alpha !} \exp k|x|<\infty .
$$

We say that $\phi_{j} \rightarrow 0$ in $\mathscr{F}(K)$ as $j \rightarrow \infty$ if there are positive constants $h$ and $k$ such that

$$
\sup _{x \in \Omega \cap \cap_{\alpha}} \frac{\left|\partial^{\alpha} \phi_{j}(x)\right|}{h^{|\alpha|} \alpha !} \exp k|x| \rightarrow 0 \text { as } j \rightarrow \infty,
$$

where $\Omega$ is any neighborhood of $K$.

We denote by $\mathscr{F}^{\prime}(K)$ the strong dual space of $\mathscr{F}^{\prime}(K)$ and call its elements Fourier hyperfunctions carried by $K$.

Definition 1.2. We say that $\phi(z)$ is in $Q(K)$ if $\phi(z)$ is holomorphic in a neighborhood of $\Omega \cap \mathbb{R}^{n}+i\{|y| \leq r\}$ for some $r>0$ and if for some $k>0$

$$
\sup _{z \in \Omega \cap \mathbb{R}^{n}+i\{|y| \leq r\}}|\phi(z)| \exp k|z|<\infty,
$$

where $\Omega$ is a neighborhood of $K$ in $\mathbb{D}^{n}$. 
Remark. Let $K$ be a compact subset of $\boldsymbol{D}^{n}$. Then for any neighborhood $\Omega$ of $K$ in $D^{n}$ there exists a neighborhood $V$ of $K$ in $D^{n}$ such that for some $\delta>0$

$$
\left(\bar{V} \cap \boldsymbol{R}^{n}\right)_{\delta} \subset \Omega \cap \boldsymbol{R}^{n},
$$

where $U_{\delta}=\left\{x \in \boldsymbol{R}^{n}|| x-y \mid \leq \delta\right\}$ for some $y \in U \subset \boldsymbol{R}^{n}$.

We denote by $E(x, t)$ the $n$-dimensional heat kernel:

$$
E(x, t)= \begin{cases}(4 \pi t)^{-n / 2} \exp \left(-|x|^{2} / 4 t\right), & t>0 \\ 0, & t \leq 0 .\end{cases}
$$

Theorem 1.3. For every $\phi \in \mathscr{F}(K)$, let

$$
\phi_{t}(x)=\int_{\boldsymbol{R}^{n_{1}}} E(x-y, t) \phi(y) d y, \quad t>0 .
$$

Then $\phi_{t} \in Q\left(\boldsymbol{D}^{n}\right)$ and $\phi_{t} \rightarrow \phi$ in $\mathscr{F}(K)$ as $t \rightarrow 0_{+}$.

Proof. Let $\phi \in \mathscr{F}(K)$. Then we can easily show that $\phi_{t}$ is in $Q\left(\boldsymbol{D}^{n}\right)$. There are positive constants $C, h, k$ and $\delta$ such that

$$
\sup _{x \in K_{\delta} \cap \boldsymbol{R}^{n}}\left|\partial^{\alpha} \phi(x)\right| \leq C h^{|\alpha|} \alpha ! \exp (-k|x|)
$$

On the other hand we have for any $\delta>0$

$$
\begin{aligned}
\partial_{x}^{\alpha}\left(\phi_{t}(x)-\phi(x)\right) & =\int_{|y| \leq \delta} E(y, t) \partial_{x}^{\alpha}(\phi(x-y)-\phi(x)) d y \\
& +\int_{|y| \geq \delta} E(y, t) \partial_{x}^{\alpha} \phi(x-y) d y \\
& -\int_{|y| \geq \delta} E(y, t) \partial_{x}^{\alpha} \phi(x) d y \\
& =I_{1}+I_{2}+I_{3} .
\end{aligned}
$$

Making use of (1.1), we have for $|y| \leq \delta$

$$
\begin{aligned}
\sup _{x \in K \cap \mathbb{R}^{n}}\left|\partial^{\alpha} \phi(x-y)-\partial^{\alpha} \phi(x)\right| \\
\quad \leq C^{\prime}|y| h^{|\alpha|+1}(|\alpha|+1) ! \exp (-k|x|) \\
\leq C^{\prime \prime}|y|(H h)^{|\alpha|}|\alpha| ! \exp (-k|x|)
\end{aligned}
$$

for some $C^{\prime}, C^{\prime \prime}$ and $H<1$.

For any $\varepsilon>0$, taking $\delta>0$ so small that $C^{\prime \prime} \delta<\varepsilon$, we have

$$
\frac{\left|I_{1}\right| \exp k|x|}{(H h)^{|\alpha|}|\alpha| !}<\varepsilon
$$


and it follows from (1.1) that

$$
\sup _{x \in K \cap \mathbb{R}^{n}} \frac{\left|I_{3}\right| \exp k|x|}{h^{|\alpha|} \alpha !} \leq C \int_{|y| \geq \delta} E(y, t) d y \rightarrow 0
$$

and

$$
\sup _{x \in K \cap \mathbb{R}^{n}} \frac{\left|I_{2}\right| \exp k|x|}{h^{|\alpha|} \alpha !} \leq C \int_{|y| \geq \delta} E(y, t) \exp k|y| d y \rightarrow 0
$$

as $t \rightarrow 0_{+}$. This completes the proof.

Theorem 1.4. $\mathscr{I}(K)$ is topologically isomorphic to $Q(K)$.

Proof. Let $\phi \in Q(K)$. Then $\phi$ is holomorphic in a neighborhood of $\Omega \cap \mathbb{R}^{n}+i\{|y| \leq r\}$ for some $r>0$ and for some $k>0$

$$
\sup _{z \in \Omega \cap \mathbb{R}^{n}+i\{|y| \leq r\}}|\phi(z)| \exp k|z|<\infty,
$$

where $\Omega$ is any neighborhood of $K$ in $\mathbb{D}^{n}$. Let $\frac{1}{r}=h>0$. Then for $x \in \Omega \cap$ $\mathbb{R}^{n}$, we have

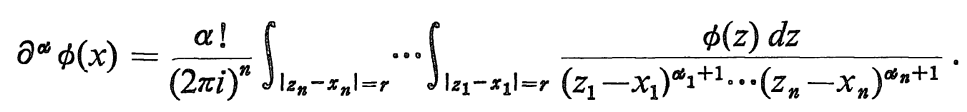

Let $z=\xi+i \eta$. If $\left|x_{j}\right| \geq 2 r$ then we have

$$
\left|\xi_{j}\right| \geq\left|x_{j}\right| / 2
$$

Therefore it follows that

$$
\begin{aligned}
\left|\partial^{\alpha} \phi(\alpha)\right| & \leq h^{|\alpha|} \alpha ! \sup _{\left|z_{j}-x_{j}\right|=r}|\phi(z)| \\
& \leq h^{|\alpha|} \alpha ! \sup _{\left|z_{j}-x_{j}\right|=r}|\phi(z)| \exp k\left(|\xi|-\frac{|x|}{2}\right) \\
& \leq C h^{|\alpha|} \alpha ! \exp (-k|x| / 2) \sup _{z \in \Omega \cap \mathbb{R}^{n}+i\{|\eta| \leq r\}}|\phi(z)| \exp k|z| .
\end{aligned}
$$

Hence we have

$$
\frac{\left|\partial^{\infty} \phi(x)\right|}{h^{|\alpha|} \alpha !} \exp k|x| / 2 \leq C \sup _{z \in \Omega \cap \mathbb{R}^{n}+i\{|\eta| \leq r\}}|\phi(z)| \exp k|z|
$$

for any $\left|x_{j}\right| \geq 2 r$.

On the other hand, we have for any $\left|x_{j}\right| \leq 2 r$

$$
\left|\partial^{\alpha} \phi(x)\right| \exp k|x| / 2
$$




$$
\leq \exp (k \sqrt{n} / h) \cdot \alpha ! h^{|\alpha|} \sup _{z \in \Omega \cap \boldsymbol{R}^{n}+i\{|\eta| \leq r\}}|\phi(z)| \exp k|z| .
$$

Therefore it follows that

$$
\begin{aligned}
& \sup _{x \in \Omega \cap \boldsymbol{R}^{n}} \frac{\left|\partial^{\alpha} \phi(x)\right|}{h^{|\alpha|} \alpha !} \exp k|x| \\
& \quad \leq \exp (2 k \sqrt{n} / h) \sup _{z \in \Omega \cap \boldsymbol{R}^{n}+i\{|\eta| \leq r\}}|\phi(z)| \exp k|z| \\
& <\infty
\end{aligned}
$$

Let $\phi \in \mathscr{F}(K)$. Then it follows from Pringsheim Theorem that $\phi$ can be analytically continued to a strip $\left\{z=x+i y\left|x \in \Omega \cap \boldsymbol{R}^{n},\right| y \mid \leq r<\frac{1}{h}\right\}$

$$
\begin{aligned}
|\phi(z)| \exp k|x| & =\exp k|x|\left|\sum_{\alpha} \frac{\partial^{\alpha} \phi(x)}{\alpha !}(i y)^{\alpha}\right| \\
& \leq C \sum_{\alpha} \frac{\left|\partial^{\alpha} \phi(x)\right|}{h^{|\alpha|} \alpha !} \exp k|x|(h|y|)^{|\alpha|} \\
& \leq C^{\prime} \sum_{\infty}(h r)^{|\alpha|} .
\end{aligned}
$$

Therefore we have

$$
\sup _{z \in \Omega \cap \boldsymbol{R}^{n}+i\{|y| \leq 1 / h\}}|\phi(z)| \exp k|z|<\infty,
$$

which completes the proof.

Remark. Let $K$ be a compact set in $\boldsymbol{D}^{n}$ and let $u \in \mathcal{F}^{\prime}(K)$. Then for any $h, k>0$ there is a constant $C$ such that

$$
|u(\phi)| \leq C \sup _{x \in \Omega \cap \boldsymbol{R}^{n}} \frac{\left|\partial^{\alpha} \phi(x)\right|}{h^{|\alpha|} \alpha !} \exp k|x|, \phi \in Q\left(\boldsymbol{D}^{n}\right),
$$

where $\Omega$ is any neighborhood of $K$ in $D^{n}$.

This is equivalent to the condition that for every neighborhood $\Omega$ of $K$ and for every $k>0$ there is a constant $C$ such that

$$
|u(\phi)| \leq C \sup _{x \in \Omega \cap \boldsymbol{R}^{n}+i\{|\eta|+r\}}|\phi(z)| \exp k|z|, \phi \in Q\left(\boldsymbol{D}^{n}\right) .
$$

Proposition 1.5. Let $P(\partial)=\sum_{|\alpha|=0}^{\infty} a_{\alpha} \partial^{\alpha}$ be a differential operator of infinite order with constant coefficients satisfying the following: For any $L>0$ there exists a constant $C>0$ such that

$$
\left|a_{\alpha}\right| \leq C L^{|\alpha|} / \alpha !
$$

for all $\alpha$. Then the operators 


$$
P(\partial): \mathscr{F}\left(\mathbb{D}^{n}\right) \rightarrow \mathscr{F}\left(\mathbb{D}^{n}\right)
$$

and

$$
P(\partial): \mathscr{F}^{\prime}\left(\mathbb{D}^{n}\right) \rightarrow \mathscr{F}^{\prime}\left(\mathbb{D}^{n}\right)
$$

are continuous.

Proof. Let $\phi \in \mathscr{F}\left(\mathbb{D}^{n}\right)$ and $h>0$. Then it follows that

$$
\begin{aligned}
\mid \partial^{\beta} & \mathbb{P}(\partial) \phi(x)|\exp k| x \mid \\
& \leq \sum_{|\alpha|=0}^{\infty}\left|a_{\alpha \mid}\right|\left|\partial^{\alpha+\beta} \phi(x)\right| \exp k|x| \\
& \leq \sum_{|\alpha|=0}^{\infty}|\phi|_{k, h} \frac{C L^{|\alpha|}}{\alpha !} h^{|\alpha+\beta|}(\alpha+\beta) ! \\
& \leq C|\phi|_{k, h}(2 h)^{|\beta|} \beta ! \sum_{|\alpha|=0}^{\infty}(2 h L)^{|\alpha|} .
\end{aligned}
$$

Thus if we choose $h>0$ so small that $2 L h<1$ then we obtain

$$
|P(\partial) \phi(x)|_{k, 2 h} \leq C|\phi|_{k, h}, \quad \phi \in \mathscr{F}\left(\mathbb{D}^{n}\right) .
$$

which proves that (1.3) is continuous. The continuity of (1.4) is easily obtained by this fact.

\section{§ 2. Main Theorems}

The following lemma is very useful later. For the details of the proof we refer to Komatsu [13], Lemma 2.9 and Lemma 2.10.

Lemma 2.1. For any $\varepsilon_{1}>0$ there exist a function $v(t) \in C_{0}^{\infty}(\mathbb{R})$ and an ultradifferential operator $P(d / d t)$ such that

$$
\begin{gathered}
\operatorname{supp} v \subset\left[0, \varepsilon_{1}\right] \\
|v(t)| \leq C \exp \left(-N^{*}(1 / t)\right), t>0 ; \\
\text { for any } h>0, \quad P(d / d t)=\sum_{k=0}^{\infty} a_{k}(d / d t)^{k},\left|a_{k}\right| \leq C_{h} h^{k} / k !^{2} ; \\
P(d / d t) v(t)=\delta+w(t) .
\end{gathered}
$$

Here $w(t) \in C_{0}^{\infty}(\mathbb{R})$, supp $w \subset\left[\varepsilon_{1} / 2, \varepsilon_{1}\right]$ and $\delta$ is a Dirac measure, and

$$
N^{*}(t)=\sup _{p} \log \frac{t^{p}}{h_{p} p !}
$$

where $h_{p}=\left(l_{1} \cdots l_{p}\right)^{-1}$ for some sequence $l_{p}$ decreasing to 0 . 
In fact. we can construct the above ultradifferential operator $P(d / d t)$ by taking

$$
P(\zeta)=(1+\zeta)^{2} \prod_{p=1}^{\infty}\left(1+\frac{l_{p} \zeta}{p^{2}}\right) .
$$

Let $K$ be a compact set in $\boldsymbol{D}^{n}$. Then we denote by $\mathscr{F}_{K}^{\text {tame }}$ the totality of $C^{\infty}$-solutions of heal equation $\left(\partial_{t}-\Delta\right) u(x, t)=0$ on $\boldsymbol{R}_{+}^{n+1}$ which satisfy the following:

For any $\varepsilon>0$, there is a constant $C$ such that

$$
|u(x, t)| \leq C \exp \left[\varepsilon\left(\frac{1}{t}+t+|x|\right)-\frac{\operatorname{dis}\left(x, K_{\delta} \cap \boldsymbol{R}^{n}\right)^{2}}{8 t}\right]
$$

in $\boldsymbol{R}_{+}^{n+1}$. Then we have:

Theorem 2.2. Let $u \in \mathscr{F}^{\prime}(K)$ and let

$$
U(x, t)=u_{y}(E(x-y, t)), \quad t>0 .
$$

Then $U(x, t) \in \mathscr{F}_{K}^{\text {tame }}$ and

$$
U(x, t) \rightarrow u \text { in } \mathscr{F}^{\prime}(K) \text { as } t \rightarrow 0_{+} .
$$

Conversely, every element in $\mathscr{F}_{K}^{\text {tame }}$ can be expressed in the form (2.3) with unique element $u \in \mathscr{F}^{\prime}(K)$.

Proof. Let $u \in \mathscr{F}^{\prime}(K)$. Then it is obvious that the function $U(x, t)$ defined by (2.3) belongs to $C^{\infty}\left(\boldsymbol{R}_{+}^{n+1}\right)$ and satisfies the heat equation on $\boldsymbol{R}_{+}^{n+1}$;

$$
\left(\partial_{t}-\Delta\right) U(x, t)=0 \text {. }
$$

If follows from (1.2) that for $t>0$

$$
\begin{aligned}
|U(x, t)| & \leq C \sup _{\substack{y \in K_{\delta} \cap \mathbb{R}^{n} \\
|\eta| \leq r}}|E(x-y-i \eta, t)| \exp k|x| \\
& \leq C \sup _{\substack{y \in K_{\delta} \cap \mathbb{R}^{n} \\
|\eta| \leq r}}(4 \pi t)^{-n / 2} \exp \left[\frac{-(1 / 2)|x-y|^{2}+8 k^{2} t^{2}+4 k t|x|+\eta^{2}}{4 t}\right] \\
& \leq C^{\prime} \exp \left[2 k^{2} t+k|x|+\frac{r^{2}}{4 t}-\frac{\operatorname{dis}\left(x, K_{\delta} \cap \boldsymbol{R}^{n}\right)^{2}}{8 t}\right]
\end{aligned}
$$

Let $\varepsilon=\max \left\{2 k^{2}, k, r^{2} / 4\right\}$. Then we obtain (2.2). Hence $U(x, t) \in \mathscr{F}_{K}^{\text {tame }}$.

Now let

$$
G(y, t)=\int_{\boldsymbol{R}^{n}} E(x-y, t) \phi(x) d x, \quad \phi \in \mathscr{F}(K) .
$$


Then by Theorem 1.3 we can easily see that

$$
G(\cdot, t) \rightarrow \phi \text { in } \mathscr{F}(K) \text { as } t \rightarrow 0_{+} \text {. }
$$

Also, we have

$$
\int_{\boldsymbol{R}^{n}} U(x, t) \phi(x) d x=u_{y}(G(y, t))
$$

by taking the limit of the Riemann sum of the left side. Then applying (2.5) to (2.6) we obtain (2.4).

Now we will prove the converse. Let $U(x, t) \in \mathscr{F}_{K}^{\text {tame }}$ and let

$$
\Omega=\left\{(x, t) \in \mathbb{R}^{n+1} ; t \neq 0 \quad \text { or } \quad x \notin K \cap \mathbb{R}^{n}\right\}
$$

Since the heat operator is hypoelliptic the condition (2.2) implies

$$
\lim _{t \rightarrow 0_{+}} \widetilde{P}\left(\partial_{t}, \partial_{x}\right) U(x, t)=0, \quad x \notin K \cap \mathbb{R}^{n}
$$

for any linear differential operator (with constant coefficients) $\widetilde{P}\left(\partial_{t}, \partial_{x}\right)$ of finite order. It follows that there is a $C^{\infty}$-function $c(x, t)$ satisfying the following:

$$
c(x, t)=U(x, t) \text { in } \boldsymbol{R}_{+}^{n+1}
$$

and

$$
c(x, t) \text {, together with all its derivatives vanishes on } \boldsymbol{Q} \backslash \boldsymbol{R}_{+}^{n+1} \text {. }
$$

The assumption (2.2) implies that $U(x, t)$ does not increase faster than exp $\left[\varepsilon\left(\frac{1}{t}+|x|\right)\right]$ as $t \rightarrow 0_{+}$. We see that there exists a Fourier hyperfunction $\psi(x, t)$ which satisfies the following:

$$
\psi=c \text { on } \Omega
$$

and

$$
\text { supp } \psi \subset \overline{\mathbb{R}_{+}^{n+1}}
$$

In fact, let functions $v, w$ and an ultradifferential operator $P(d / d t)$ be as in Lemma 2.1. Define

$$
\tilde{c}(x, t)=\int_{0}^{\infty} c(x, t+s) v(s) d s .
$$

Then we have

$$
\left(\partial_{t}-\Delta\right) \tilde{c}(x, t)=0 \text { in } \boldsymbol{R}_{+}^{n+1} .
$$

It follows from Lemma 2.1 and (2.2) that 


$$
|\tilde{c}(x, t)| \leq C^{\prime} \exp \varepsilon(|x|+t), \quad t \geq 0 \text {. }
$$

Thus $\tilde{c}(x, t)$ is a continuous function of an infra-exponential type in $\overline{\boldsymbol{R}_{+}^{n+1}}$. Using (2.1) we obtain for $t>0$.

$$
\begin{aligned}
P(-\Delta) \tilde{c}(x, t) & =P(-d / d t) \tilde{c}(x, t) \\
& =c(x, t)+\int_{0}^{\infty} c(x, t+s) w(s) d s .
\end{aligned}
$$

Since $\tilde{c}(x, t)$ and the second term of the right hand side of (2.7) can be continuously extended beyond the hyperplane $t=0$, we obtain the extension $\psi(x, t)$ of $c(x, t)$.

Since $c(x, t)$ is a $C^{\infty}$-solution of heat equation on $\Omega$, we have

$$
\left(\partial_{t}-\Delta\right) \psi(x, t)=0 \text { on } \Omega \text {. }
$$

In what follows, a Fourier hyperfunction $\psi(x, t)$ thus obtained shall be called a Fourier tame extension of $U$ for short.

Let $g(x)=\tilde{c}(x, 0)$ and $h(x)=-\int_{0}^{\infty} c(x, s) w(s) d s$. Then $g$ and $h$ are also continuous functions of an infra-exponential type, and hence Fourier hyperfunctions. We define a Fourier hyperfunction $u$ as

$$
u(x)=P(-\Delta) g(x)+h(x) .
$$

Since

$$
\lim _{t \rightarrow 0_{\perp}} U(x, t)=0, x \notin K,
$$

we see that $u \in \mathscr{F}^{\prime}(K)$.

We define a Fourier hyperfunction $\alpha(x, t)$ by

$$
\alpha(x, t)= \begin{cases}\int E(x-y, t) u(y) d y, & t>0 \\ 0, & t<0 .\end{cases}
$$

Let $\alpha_{+}(x, t)$ be the restriction of $\alpha$ to $\boldsymbol{R}_{+}^{n+1}$. Then we have $\alpha_{+}(x, t) \in \mathscr{F}_{K}^{\text {tame }}$.

Let $\beta(x, t)$ be a Fourier tame extension of $\alpha_{+}(x, t)$. Note that $\beta$ does not coincide with $\alpha$ in general. It follows from (2.4) that

$$
\begin{aligned}
\lim _{t \rightarrow 0_{+}} \alpha(x, t) & =\lim _{t \rightarrow 0_{+}} \beta(x, t) \\
& =\lim _{t \rightarrow 0_{+}} \psi(x, t) .
\end{aligned}
$$

Hence we have

$$
\left(\partial_{t}-\Delta\right)(\psi(x, t)-\alpha(x, t))=0 \text { in } \boldsymbol{R}^{n+1}
$$


By the well known uniqueness theorem for the solutions of the Cauchy problem to the heat equation we have $\psi=\alpha$ (See [4]).

Remarks. (i) The estimate (2.2) for $K=\mathbb{D}^{n}$ is the following:

$$
|u(x, t)| \leq C \exp \varepsilon\left[\frac{1}{t}+t+|x|\right] .
$$

(ii) If $K \subset \subset \mathbb{R}^{n}$ then the estimate (2.2) is replaced by

$$
|u(x, t)| \leq C \exp \left[\frac{\varepsilon}{t}-\frac{\operatorname{dis}(x, K)^{2}}{4 t}\right] .
$$

In this case, T. Kawai and T. Matsuzawa have shown in $[9,14]$ that its boundary value determines a unique hyperfunction with carrier $K$ so that the vanishing of $g(x)$ implies the vanishing of $u(x, t)$.

(iii) Since it suffices to consider the estimate (2.2) for sufficiently small $t>0$, we may omit the term $\varepsilon t$ in (2.2).

Corollary 2.3. There exists an isomorphism

$$
b: \mathscr{F}_{K}^{\text {tame }} \rightarrow \mathscr{F}^{\prime}(K) .
$$

From the proof of Theorem 2.2, we obtain the following corollaries:

Corollary 2.4. Each function in $\mathscr{F}_{K}^{\text {tame }}$ is real analytic.

Corollary 2.5. If $u \in \mathscr{F}^{\prime}(K)$, then there exist an ultradifferential operator $P(d / d t)$ of Gevrey order 2, a continuous function $g$ of an infra-exponential type and $a C^{\infty}$-function $h$ of an infra-exponential type such that

$$
u(x)=P(-\Delta) g(x)+h(x),
$$

where $g \in C^{\infty}\left(\boldsymbol{R}^{n} \backslash K\right)$.

We can consider $\mathscr{F}^{\prime}\left(K_{1}\right) \subset \mathscr{F}^{\prime}\left(K_{2}\right)$ if $K_{1} \subset K_{2} \subset \subset \mathbb{D}^{n}$. Let $\mathscr{F}^{\prime}=\bigcup_{K \subset \subset \subset_{D^{n}}} \mathscr{F}^{\prime}(K)$. Then we have:

Theorem 2.6. If $u \in \mathscr{F}^{\prime}$ then there is a smallest compact set $K \subset \subset \mathbb{D}^{n}$ such that $u \in \mathscr{F}^{\prime}(K)$.

Proof. Let $u \in \mathscr{F}^{\prime}$ and let $K$ be the intersection of all compact set $K^{\prime}$ in $D^{n}$ such that $u \in \mathscr{F}^{\prime}\left(K^{\prime}\right)$. By Theorem 2.2 a defining function

$$
U(x, t)=u_{y}(E(x-y, t)), \quad t>0
$$

is uniquely defined and satisfies the heat equation in $\mathbb{R}^{n+1} \backslash(K \times\{0\})$. Noting 
that $u=\lim _{t \rightarrow 0_{+}} U(\cdot, t)$, we see that $u \in \mathscr{F}^{\prime}(K)$.

Theorem 2.7. Let $K_{1}, \cdots, K_{r}$ be compact subsets of $\boldsymbol{D}^{n}$ and $u \in \mathscr{F}^{\prime}\left(K_{1} \cup \cdots\right.$ $\left.\cup K_{r}\right)$. Then we can find $u_{j} \in \mathscr{F}^{\prime}\left(K_{1}\right)$ so that $u=u_{1}+\cdots+u_{r}$.

Proof. It is sufficient to prove the statement when $r=2$. Let $U(x, t)$ be the function defined by (2.3). The theorem will be proved if we can split $U$ into a sum $U=U_{1}+U_{2}$ where $U_{j} \in \mathscr{F}_{K_{j}}^{\text {tame }}, j=1,2$. Let $\tilde{U}$ be a Fourier tame extension of $U$. Then $\widetilde{U}$ satisfies the heat equation in $\boldsymbol{R}^{n+1} \backslash\left(\tilde{K}_{1} \cup \widetilde{K}_{2}\right)$ where $\tilde{K}_{j}=K_{j} \times\{0\} \cap \boldsymbol{R}^{n+1}, j=1,2$. We take a function $\psi \in C^{\infty}\left(\boldsymbol{R}^{n+1} \backslash \widetilde{K}_{1} \cap \widetilde{K}_{2}\right)$ constructed in [7, Corollary 1.4.11] such that $\psi=0$ for large $|x|+t$ and near $\left(\tilde{K}_{2} \backslash\left(\tilde{K}_{1} \cap \tilde{K}_{2}\right)\right), \psi=1$ near $\left(\tilde{K}_{1} \backslash \tilde{K}_{1} \cap \tilde{K}_{2}\right)$ and $\psi \in L^{\infty}\left(\boldsymbol{R}^{n+1}\right)$. Here "near" means in the sense of the slowly varying metric defined in [6, Chap. 1]. We will split $\widetilde{U}$ as follows:

$$
\tilde{U}_{1}=\psi \tilde{U}-\tilde{V}, \quad \tilde{U}_{2}=(1-\psi) \tilde{U}+\tilde{V} .
$$

We define $\psi \widetilde{U} \in \mathscr{F}^{\prime}\left(D^{n+1}\right)$ such that $\psi \widetilde{U}=0$ near $\left(\tilde{K}_{2} \backslash\left(\tilde{K}_{1} \cap \widetilde{K}_{2}\right)\right)$ and $(1-\psi) \widetilde{U}=0$ near $\left(\tilde{K}_{1} \backslash\left(\tilde{K}_{1} \cap \tilde{K}_{2}\right)\right)$. We can write

$$
\left(\partial_{i}-\Delta\right) \psi \widetilde{U}=\widetilde{F}+f
$$

where $\widetilde{F}$ and $f$ are in $\mathscr{F}^{\prime}\left(\boldsymbol{D}^{n+1}\right)$ such that

$$
\widetilde{F}= \begin{cases}\left(\partial_{t}-\Delta\right)(\psi U), & t>0 \\ 0, & t<0\end{cases}
$$

and $f \in \mathscr{F}^{\prime}\left(\boldsymbol{D}^{n+1}\right)$, supp $f \subset K_{1} \times\{0\}$. Now we define

$$
\tilde{V}(x, t)=E * \widetilde{F}(x, t) \in \mathscr{F}^{\prime}\left(\boldsymbol{D}^{n+1}\right)
$$

and $V(x, t) \equiv \tilde{V}(x, t)$ for $t>0$. Then we have

$$
\tilde{V} \in C^{\infty}\left(\boldsymbol{R}^{n+1} \backslash \tilde{K}_{1} \cap \tilde{K}_{2}\right), \quad \operatorname{supp} \tilde{V} \subset \overline{\boldsymbol{R}_{+}^{n+1}}
$$

and

$$
V(\cdot, t) \rightarrow 0 \text { uniformly in }\left\{x ; \operatorname{dis}\left(x, K_{1} \cap K_{2}\right) \geq \delta\right\}
$$

for every $\delta>0$ as $t \rightarrow 0_{+}$. Since we have

$$
V(x, t)=\psi U-E * f(x . t),
$$

we have for any $\varepsilon>0$

$$
V(x, t)=O(\exp \varepsilon[1 / t+t+|x|]) \text { as } t \rightarrow 0_{+} .
$$


Thus we have the desired property that

$$
U_{1}=\psi U-V \in \mathscr{F}_{K_{1}}^{\text {tame }}
$$

and

$$
U_{2}=(1-\psi) U+V \in \mathscr{F}_{K_{2}}^{\text {tame }}
$$

\section{Acknowledgement}

The authors are grateful to Professor A. Kaneko for suggesting the problem and to the referee for correcting Lemma 2.1 .

\section{References}

[1] Chou, C.C., La transformation de Fourier complexe et l'equation de convolution, Springer-Verlag, Berlin-New York, 1973.

[2] Chung, S.Y. and Kim. D., Structure of the Fourier hyperfunctions of maximal type, preprint.

[3] —, Equivalence of the defining sequences for ultradistributions, to appear in Proc. Amer. Math. Soc.

[4] - Uniqueness for the Cauchy problem of heat equations without uniform condition on time, preprint.

[5] Chung, S.Y., Kim, D. and Kim, S.K., Solvability of Mizohata and Lewy operators, to appear in J. Fac. Sci. Univ. Tokyo, Sect. IA, 40 (1993).

[6] Gel'fand, I.M. and Shilov, G.E., Generalized functions III, Academic Press, New York and London, 1967.

[7] Hörmander, L., The analysis of linear partial differential operator I, Springer-Verlag, Berlin-New York, 1983.

[8] - Between distributions and hyperfunctions, Astérisque, 甚1 (1985), 89-106.

[9] Kaneko, A., Introduction to hyperfunctions, KIT Sci. Publ., 1988.

[10] Kawai, T. and Matsuzawa, T., On the boundary value of a solution of the heat equation, Publ. RIMS. Kyoto Univ., 25 (1989), 491-498.

[11] Komatsu, H., Ultradistributions I, J. Fac. Sci. Univ. Tokyo, Sect. IA, 20 (1973), 25-105.

[12] - Ultradistributions II, J. Fac. Sci. Univ. Tokyo. Sect. IA, 24 (1977), 637-628.

[13] - Introduction to the theory of generalized functions, Iwanami Shoten, Tokyo, 1978, (in Japanese).

[14] Matsuzawa, T., A calculus approach to hyperfunctions I, Nagoya Math. J., 108 (1987), 53-66.

[15] - A calculus approach to hyperfunctions II, Trans. Amer. Math. Soc., 313 (1989), 619-654.

[16] - A calculus approach to hyperfunctions III, Nagoya Math. J., 118(1990), 133153.

[17] Seeley, R.T., Extension of $C^{\infty}$-functions defined in a half space, Proc. Amer. Math. Soc., 15 (1964), 625-626.

[18] Widder, D.V., The heat equation, Academic Press, 1975. 